\title{
L'histoire des sciences et des techniques dans cinq dispositifs innovants de formation professionnelle : une analyse comparative
}

\section{Alain Bernard et Loïc Petitgirard}

\section{OpenEdition}

Journals

Édition électronique

URL : http://journals.openedition.org/trema/3748

DOI : $10.4000 /$ trema.3748

ISSN : 2107-0997

Éditeur

Faculté d'Éducation de l'université de Montpellier

Édition imprimée

Date de publication : 1 mars 2018

Pagination : 1 - 16

ISBN : 979-10-96627-04-2

ISSN : 1167-315X

\section{Référence électronique}

Alain Bernard et Loïc Petitgirard, «L'histoire des sciences et des techniques dans cinq dispositifs

innovants de formation professionnelle : une analyse comparative ", Tréma [En ligne], 48 | 2018, mis en ligne le 01 juin 2018, consulté le 01 mai 2019. URL : http://journals.openedition.org/trema/3748 ;

DOI : 10.4000/trema.3748

Ce document a été généré automatiquement le 1 mai 2019.

Trema 


\title{
L'histoire des sciences et des techniques dans cinq dispositifs innovants de formation professionnelle : une analyse comparative
}

\author{
Alain Bernard et Loïc Petitgirard
}

\section{Introduction : contexte et enjeux de cette comparaison critique}

1 Ce numéro thématique se fonde sur l'examen comparatif de cinq dispositifs de formation professionnelle développés dans des contextes et pour des publics a priori très différents : deux ateliers développés au Conservatoire National des arts et métiers (CNAM) en lien avec une formation pour médiateurs scientifiques, l'Atelier de médiation numérique (AMN) piloté par Loïc Petitgirard, d'une part, et un atelier mobilisant « la culture ordinaire des sciences et techniques » en lien au dispositif La Lucarne, piloté par Michel Letté d'autre part; le projet MEDFILM développé à l'université de Strasbourg par Christian Bonah et ses collègues, en lien à plusieurs cours associés dont des formations professionnelles pour médecins ; un groupe « histoire et épistémologie » expérimenté depuis 2015-16 par Alain Bernard, Katalin Gosztonyi à l'IREM de Paris Nord en partenariat avec l'École Supérieure du Professorat et de l'Éducation (ESPE) de Créteil, pour des enseignants en formation initiale ou continue; enfin le dispositif éditorial mis en place par Muriel Guedj et ses collègues à l'université de Montpellier pour accompagner l'exposition « Regards croisés sur la lumière " organisée localement à l'occasion de l'Année Internationale de la Lumière, et qui implique des élèves enseignants pour le professorat des écoles. En complément, un article (qui peut être perçu comme un épilogue), rédigé par Lisa Chupin, 
propose une relecture de l'ensemble des discussions à la lumière de sa propre étude du fonctionnement d'un herbier numérique collaboratif et citoyen, Les herbonautes.

Malgré la diversité évidente des contextes institutionnels, des publics d'étudiants et des professionnels concernés, des objets ou des objectifs de ces dispositifs, ils partagent des points communs que nous mettrons ici en évidence. A minima, tous en effet combinent, d'une manière ou d'une autre, des objectifs de formation professionnelle, des démarches de recherche en histoire des sciences et des techniques sur des objets variés, enfin des dispositifs dialogiques permettant la confrontation réflexive des étudiants ou stagiaires entre eux et avec des experts. Dans la seconde partie, nous insistons sur la variété des contextes de formation ainsi mis en regard les uns des autres, sur l'intérêt de ce type de comparaison, et sur ses limites. Ces parties introductives peuvent être regardées comme un avant-propos de ce numéro thématique.

3 Les troisième et quatrième parties nous permettent d'approfondir la comparaison et constituent le cœur de l'article. Tout d'abord, nous discutons de la façon dont s'articulent, dans chacun d'entre eux, les enjeux de recherche et de formation, en quoi ils constituent pour les professionnels concernés des espaces d'intéressement (Legros, 2008) où les pratiques collaboratives et dialogiques tiennent une place centrale (partie III). Nous récapitulons, dans la quatrième partie, comment l'élaboration raisonnée de ces différents dispositifs s'appuie sur le détournement de concepts et de problématiques théoriques, partagés par tout ou partie des auteurs des différentes contributions.

4 L'article de L. Chupin peut être regardé comme un contrepoint à ces approches théoriques: fondé quant à lui sur l'approche des sciences de l'information et de la communication, il pointe la fonction des espaces d'écriture et de collaboration numérique dans certains des dispositifs discutés.

5 La "problématisation comparative ", qui est au cœur de notre propos, est directement issue de deux journées d'étude organisées au CNAM en 2015 (Paloque-Berges 2015; Bernard 2014 et 2015) puis 2016 (Paloque-Berges 2016) et qui ont impliqué l'ensemble des co-auteurs. L'enjeu de ces rencontres était à la fois pratique et théorique : d'un point de vue pratique, il s'agissait d'enrichir et renforcer chaque dispositif par la comparaison et la mutualisation de pratiques; d'un point de vue théorique, il s'agissait de se donner les moyens de penser les enjeux fondamentaux communs à ces dispositifs. Cette dialectique nous permet jusqu'à aujourd'hui de consolider nos dispositifs ou d'en inventer de nouveaux.

6 En général, et à l'imitation des manifestations et publications évoquées ci-dessus, nous espérons que cette discussion critique et comparative pourra inspirer des lecteurs engagés dans des aventures pédagogiques similaires. L'intérêt pratique qu'ils y trouveront sera d'interroger et d'améliorer leur pratique, en fonction de la comparaison à des dispositifs différents mais similaires. L'intérêt théorique qu'ils pourront y trouver dérive du fait que plusieurs des concepts qu'on peut mobiliser pour comprendre et comparer ces dispositifs, s'avèrent largement transversaux : c'est ce qu'on verra dans les deux dernières parties, qui résument le dialogue entre les contributions. 


\section{Des contextes de recherche et de formation variés, propices à la comparaison critique}

7 Revenons tout d'abord à la variété des contextes et publics concernés et aux objectifs de formation professionnelle sous-jacents : ce qui les rapproche et autorise la comparaison, mais aussi ce qui la limite. Ces éléments de circonstance ne doivent pas en effet être oubliés alors même que nous visons des théorisations communes (parties III et IV).

\section{1. Trois contextes de formation professionnelle, qui font tous l'objet de réflexions critiques à l'échelon national : médecine, médiation et enseignement scientifique}

8 La contribution de $\mathrm{C}$. Bonah et $\mathrm{J}$. Danet s'intéresse à la formation de futurs médecins en sciences humaines, de la deuxième à la cinquième année, c'est-à-dire dans la période où ils sont déjà en situation d'alternance. Même si le public d'étudiants concerné par la plateforme MEDFILM est considérablement plus large que celui qui fait ici l'objet de leur attention (Bonah et Danet, 2014), ce dernier a une importance stratégique car c'est d'abord pour ces étudiants que la plateforme a été conçue : la genèse même de MEDFILM, et ce qui reste encore aujourd'hui sa raison d'être, s'ancre dans une pratique pédagogique ciblée sur le développement professionnel des médecins. Comme le rappellent les auteurs, les réflexions portant en général sur les enseignements de sciences humaines dans ce secteur de formation professionnelle, dépendent d'un contexte institutionnel favorable depuis les années 90. Ils font en outre l'objet à l'échelon national de recherches et de réflexions stratégiques au sein d'une association d'enseignement dédiée.

Les contributions de M. Letté et L. Petitgirard sont ciblées sur un public de médiateurs scientifiques d'âges et de profils très variés, et qui bénéficient des formations dispensées au CNAM : il s'agit donc d'un dispositif de formation continue dans le cadre institutionnel très structuré $\mathrm{du}$ Conservatoire, qui a ses traditions propres. Ce secteur professionnel a connu un développement récent voire très récent, sous l'effet d'un double mouvement du développement d'une politique publique visant à la diffusion de la «culture scientifique et technique " d'une part, et des démarches associatives qui, dès les années 1970, ont critiqué ce modèle diffusionniste et « technocratique » et proposé des modèles alternatifs plus proches de la culture populaire (Las Vergnas 2011, et les contributions de M. Letté et L. Petitgirard dans ce volume). Comme le rappelle L. Petitgirard, la réflexion critique autour du développement de ces formations, leur impact sociétal et leur rapport aux sciences humaines contributrices, fait l'objet d'investissements et de forums nationaux qui traduisent là aussi l'importance politique et stratégique accordée aujourd'hui à ce secteur.

10 Enfin les contributions d'A. Bernard, K. Gosztonyi d'une part, et de M. Guedj d'autre part, ciblent un public d'étudiants et de professionnels dont la formation et la professionnalité sont en plein renouvellement, comme en témoignent deux réformes majeures dans les huit dernières années: celui des enseignants du premier ou du second degré. La formation en histoire des sciences et des techniques de ce public ne fait pas l'objet, au niveau national, de réflexions de collectifs pérennes structurés, malgré le soutien ancien de sociétés savantes comme la SFHST ${ }^{1}$ ou bien d'éphémères tentatives comme le collectif 
ReForEHST $^{2}$ entre 2005 et 2009. Dans des disciplines particulières comme les mathématiques, par contre, il en existe depuis les années 1970, en l'espèce des travaux de la commission inter-IREM « Histoire et épistémologie ». Par ailleurs le dispositif présenté par M. Guedj concerne en réalité un public d'étudiants nettement plus large que les futurs enseignants qui font l'objet de son attention : le dispositif global associé à l'exposition « Regards croisés sur la lumière » implique des médiateurs en formation, des étudiants en histoire des sciences, ainsi qu'une grande variété de professionnels. Enfin le développement des ESPE et de leur réseau, depuis 2013, a progressivement recréé les conditions pour conduire des réflexions collectives sur la place de la recherche dans la professionnalité enseignante (Bernard, 2016a).

\section{2. Les caractéristiques communes des dispositifs discutés}

11 Au titre de ce qui autorise la comparaison entre ces divers contextes de formation, audelà de la variété des contextes, notons tout d'abord qu'il s'agit dans tous les cas de former des professionnels en tenant fondamentalement compte de leur expérience professionnelle et du terrain qui est propre à ce dernier : l'hôpital pour les médecins, les musées et centres de culture scientifique et technique pour les médiateurs, les écoles, collèges ou lycées pour les enseignants. Cette intégration de l'expérience à la formation a des racines politiques et scientifiques très profondes, comme le rappellent $\mathrm{M}$. Tardif et ses collègues dans leurs réflexions rétrospectives sur la diffusion du modèle $d u$ " praticien réflexif » (Tardif, Borges et Malo, 2012) - question sur laquelle nous reviendrons en partie IV. Cette orientation se traduit concrètement par l'existence de référentiels de compétences, ou du moins d'instances qui proposent une définition des compétences cibles des professionnels concernés. Parmi ces dernières, le rapport régulateur accordé à la recherche dans le développement de la réflexion professionnelle tient généralement une place importante : c'est une question par exemple très centrale dans la constitution des ESPE pour les enseignants ${ }^{3}$.

12 Corrélativement, les dispositifs qui sont ici discutés, à l'exemple du design thinking (Aillerie, 2015 ; Brown, 2014) qui inspire le dispositif discuté par L. Petitgirard, s'inscrivent par la force des choses dans les réflexions sur la pédagogie dans l'enseignement supérieur, qui s'imposent progressivement dans le contexte français. Cela veut dire concrètement qu'une attention particulière est portée à l'alignement stratégique entre les objectifs de formation, ses supports et outils, et les questions d'évaluation. Cette dimension est d'autant plus importante pour nous ici, que les compétences visées sont pour partie d'un ordre très élevé : il s'agit de développer au minimum une capacité à une réflexivité autonome sur les pratiques, mais aussi des compétences en histoire et l'épistémologie des sciences et techniques ou portant en général sur l'étude des sciences en société, enfin dans plusieurs cas à une forme de créativité dans l'invention de dispositifs d'enseignement et/ou de médiation.

En outre et comme on l'a souligné plus haut, tous ces dispositifs ont en commun de pouvoir s'appuyer, selon le corps de discipline ou le secteur professionnel concerné, sur des instances de régulation à l'échelon national: mutualisation des démarches, recherches ou au moins réflexions critiques sur la formation en histoire des sciences ou plus généralement en sciences humaines. De manière plus transversale, et puisqu'à chaque fois les dispositifs se rattachent aux recherches en histoire et sociologie des sciences et des techniques, ils ont en commun, précisément, un horizon théorique 
général, issu du développement traditionnel de l'épistémologie historique, et plus récemment du courant des "sciences et techniques en société " (science and technology studies) $)^{4}$.

\section{3. Les potentialités de la comparaison, au-delà des limites évidentes}

Mais les points communs soulignés ci-dessus ne représentent pas vraiment, ou pas toujours, de réels points de convergence, précisément parce que les secteurs professionnels, ou les instances de régulation correspondantes, sont largement cloisonnés entre eux. Même les instances de régulation transversales, malgré les titres communs, masquent mal cette diversité d'approches et de contextes. Quant au rapprochement de ces secteurs sur la base des compétences qui s'y développent, il se heurte aux critiques adressées à une approche décontextualisée des compétences (Rey 2014), qui fait oublier que leur exercice dépend des particularités des contextes professionnels et savoirs ou savoir-faire sous-jacents, ou encore des objets à valeur patrimoniale et historique étudiés.

Il faut à ce titre souligner l'originalité précieuse des journées d'étude évoquées en introduction (Paloque-Berges, 2015, 2016), et qui ont rendu possible ce volume. Plus en amont, deux évènements et rencontres ont posé des jalons préliminaires : le colloque interdisciplinaire et interprofessionnel organisé à l'UPEC en 2013 (Bernard et al. 2014) dans le sillage duquel ces journées se sont inscrites; et la série de séminaires au CNAM (dans le cadre du Labex HASTEC ${ }^{5}$, depuis 2013) autour des "Légitimations du savoir : le rôle des techniques dans la construction sociale des savoirs légitimes", lesquels ont contribué à réunir des acteurs académiques et professionnels, d'horizons très divers, interdisciplinaires, et qui n'ont que très peu d'interactions habituellement. Ces manifestations rendent possible un dialogue que les institutions régulatrices auxquelles on a fait allusion plus haut, ne permettent pas concrètement d'installer, ou seulement marginalement.

\section{Un enjeu commun : ouvrir et élaborer des espaces de réflexion}

Nous nous intéressons ici à deux points communs évidents de l'ensemble des dispositifs de formation discutés dans ce volume: le fait qu'ils se donnent tous pour ambition de ménager, à chaque fois selon des modalités et stratégies pédagogiques différentes, des espaces de réflexion sur l'activité professionnelle; et que les stratégies d'ouverture de ces espaces s'appuient en partie sur une expertise en histoire des sciences et des techniques, dans un sens large incluant les perspectives d'histoire culturelle et des domaines en lien avec le champ des "sciences, techniques et société ». Sur la base de ce constat nous proposons dans cette partie quelques éléments de comparaison critique (III.I), permettant de mieux comprendre des éléments de convergence plus profonds (III.2 et III.3). 


\section{1. Deux grandes stratégies pédagogiques : créer pour réfléchir, ou réfléchir pour créer}

17 En termes stratégiques, il est aisé de proposer une première différence entre des dispositifs, comme ceux décrits par M. Guedj ou L. Petitgirard, qui se fondent sur une pédagogie par projet $^{6}$. Le développement d'une réflexion professionnelle vient se greffer comme un élément ou une étape du processus d'invention collective de ces dispositifs. On pourrait donc dire qu'il s'agit de dispositif du type «créer pour réfléchir» (créer-pourréfléchir ou $\mathrm{CpR}$ dans la suite), où on met d'abord l'accent sur l'invention, le design. $\mathrm{A}$ contrario, les dispositifs de formation ou d'accompagnement professionnel développés par C. Bonah et J. Danet d'un côté, A. Bernard et K. Gosztonyi de l'autre, se fondent sur une démarche réflexive dont ils cherchent à définir les catalyseurs, films documentaires dans un cas, textes sériels de problèmes dans l'autre. Le processus transitionnel recherché doit permettre aux participants de développer une forme de créativité professionnelle - et, dans le cas des étudiants médecins, une forme de savoir être. On peut donc parler d'un type de dispositif conduisant à « réfléchir pour (re)créer » (réfléchir-pourcréer ou RpC dans la suite), où l'accent principal porte cette fois-ci sur la réflexion presque dans un sens premier : les objets proposés fonctionnent comme des miroirs de l'expérience professionnelle et permettent d'en élaborer de nouvelles représentations susceptibles de contribuer à son enrichissement. Quelque part entre les deux, la proposition de $\mathrm{M}$. Letté s'apparente au premier type parce qu'elle se situe dans un contexte de formation qui vise fondamentalement à inventer de nouvelles formes de médiation (le public est le même que celui des Ateliers de médiation numériques de L. Petitgirard) ; mais le point d'entrée est une démarche réflexive qui s'ancre dans un acte de reconnaissance du caractère omniprésent et déjà médiatisé de la culture scientifique. Il articule une heuristique de la culture ordinaire des sciences et société avec un dispositif de type réfléchir-pour-créer (l'atelier La Lucarne). Si on suit la logique ci-dessus proposée de construction d'une typologie, il faudrait ajouter ici une instance médiane comme "réfléchir à du créé, pour recréer» (RCpC), qui serait une variante des dispositifs réfléchir-pour-créer.

18 Ce qu'indique ce cas " médian » ne lui est probablement pas particulier : après tout, les dispositifs de M. Guedj ou L. Petitgirard se fondent tous deux sur une phase pré-réflexive construite sur un choix argumenté de dispositifs, qu'il s'agit de recréer : premières visites de l'exposition dans le cas de M. Guedj, enquête préliminaire de type ethnographique dans le cas de L. Petitgirard. En général, les dispositifs comparés ont en commun de conjuguer réflexivité professionnelle et créativité dans plusieurs étapes clés, et à cet égard la typologie ci-dessus n'indique que de simples différences d'emphase et d'ordre stratégique. Son principal avantage est qu'elle permet de repérer ce qui se rapproche ou s'éloigne plus ou moins d'un modèle de pédagogie par projet.

Cette typologie de dispositifs de formation est axée fondamentalement sur ce qui en fait des dispositifs de formation initiale ou continue, autrement dit sur les aspects qui favorisent une forme de réflexivité à valeur formatrice. Mais on peut aussi les regarder autrement, à partir des formes de mobilisation d'objets de collections ou de la culture scientifique ainsi que d'outils numériques d'écriture et de publication, selon les étapes du processus dans lesquelles elles interviennent, et les finalités qu'elles servent à atteindre. Ce point de vue est développé tout particulièrement dans l'article de L. Chupin. 


\section{2. La place dévolue à l'expertise en histoire et épistémologie des sciences}

20

Dans les différentes approches stratégiques relevées ci-dessus, la place de l'expertise en sciences humaines (histoire et philosophie des sciences et techniques, approches "STS » des sciences et techniques en société) joue à deux niveaux fondamentaux, celui de la définition et de l'interprétation des objets et supports de réflexion d'une part; celui de la posture de recherche d'autre part.

dispositifs creer-pour-réfléchir, il s'agit de s'approprier des dispositifs d'interprétation ou de valorisation des objets visés (patrimoniaux, historiques), et cette appropriation s'appuie en particulier sur une forme de contextualisation savante. L'appropriation vise en outre à donner la possibilité d'un détournement, d'une recréation. Dans le cas du dispositif décrit par M. Guedj, l'expertise historienne entre une première fois en jeu dans la négociation experte qui organise la conception de l'exposition servant de cadre général à la démarche pédagogique discutée, sous la forme classique d'une "biographie savante» des objets de l'exposition et du contexte sociohistorique qui leur donne sens. Mais les étudiants concernés doivent en quelque sorte se réapproprier une partie de cette expertise, sur les objets qu'ils choisissent : c'est ce qui résulte du cadrage qui leur est imposé. La régulation experte joue ici une seconde fois, cette fois-ci pour guider un ajustement des premiers choix d'objets qui donne une réelle cohérence à la fois scientifique, historique et probablement esthétique à ces choix. Autrement dit, c'est une partie de la démarche historienne que les étudiants sont invités à récupérer, en s'appuyant sur les éléments fournis par le travail de conception de l'exposition transporté par l'objet frontière ${ }^{7}$ qui a servi à la structurer, et disponibles via les enseignants. Cette problématique du recours à une expertise se retrouve explicitement dans le dispositif de l'Atelier de médiation numérique problématisé par L. Petitgirard, sauf que la comparaison révèle que, dans ce cas, les étudiants ne disposent pas d'un équivalent du document structurant l'exposition : au mieux, ils doivent reconstituer les enjeux de l'action muséographique qui entoure les objets de leur choix, par une enquête préalable. C'est une double expertise qui est mobilisée : une analyse de type STS centrée sur les enjeux médiatiques et muséographiques d'une part, une expertise historienne d'autre part. La première accompagne l'appropriation du dispositif existant en vue de son détournement et de sa recréation. La seconde vise l'appropriation des objets des collections dans leur épaisseur socio-historique, d'autant plus importante qu'ils sont au cœur du dispositif de médiation existant et leur (futur) prototype de médiation numérique. Dans les deux cas, l'intégration de cette expertise passe par les phases dialogiques et de confrontations aux experts (enseignants, historiens, professionnels de la médiation). Dans le cas de l'Atelier de médiation numérique, il est en outre appuyé par un processus d'écriture réflexive des étudiants assurant la négociation des dissensus internes et la structuration du travail collectif.

Dans le cas des dispositifs réfléchir-pour-créer et RCpC, l'expertise historienne, STS, ou également cinématographique (dans le cas Bonah-Danet) a plutôt, au départ, un rôle «étrangéisant»: elle met en crise la familiarité acquise avec un objet auquel les participants se sont d'abord attachés, ou avec leurs représentations habituelles de la médiation. C'est l'appropriation qui prime et l'expertise donne les moyens aux participants de différencier ce qui ressort d'une projection de l'expérience, de la nature

Tréma, 48 | 2018 
construite de l'objet. En outre et dans la seconde phase du travail, cette expertise est mise au service de l'étude de nouveaux objets, laissés au choix des étudiants. Dans le cas de M. Letté, ce choix porte sur des objets et des documents relevant d'une manifestation ordinaire des sciences et techniques en société. Les reconnaître comme construits selon une sorte de "médiation naturelle » ou " déjà là » relève d'une expertise STS comme de ce qui permet de développer cette analyse, légitimant ainsi cette expertise. Nous revenons sur cette question de la manière de comprendre les objets mis en circulation et transformés dans ces dispositifs, dans la partie suivante, et L. Chupin en propose une interprétation complémentaire, à partir de la notion de trivialité, dans son épilogue ( $\mathrm{p}$. 121).

$\mathrm{Au}$ niveau de la posture de recherche, il apparaît que les experts-formateurs qui interviennent dans tous les dispositifs décrits ici, s'appuient tout autant sur leur posture de formateurs que sur leur savoir de chercheurs. Car l'expertise peut être simplement vécue et ressentie par les participants et étudiants comme une barrière, un verrou à leurs propres interprétations et re-créations. L'autorité d'un savoir acquis sur les objets s'impose alors, à tort ou à raison, comme seule interprétation possible de ces derniers, et comme une forme de possession.

Ce problème est assez aisément réglé dans les dispositifs créer pour réfléchir, précisément parce qu'ici l'invention et la recréation priment : l'expertise peut alors être mise en jeu à titre critique, ou à titre de régulation comme on l'a vu plus haut, mais elle ne dépossède pas les participants de leur créativité. Dans les dispositifs réfléchir-pour-créer, le fait d'introduire des objets filmiques ou textuels (ou les deux, comme dans le cas de M. Letté et en particulier dans l'atelier «la Lucarne ») comme objets de questionnement tout autant que d'expérience, y compris pour les enseignants, joue un rôle fondamental pour le dialogue collectif qui s'instaure.

Ce rôle est celui d'une précondition: il permet au dialogue de s'instaurer, sans être verrouillé par une interprétation déjà faite. Lors de la journée d'étude de mars 2016, C. Bonah a décrit de manière frappante, comme une alternative possible à la diffusion de films documentaires de la base MEDFILM, celle d'enregistrements récents de consultations des enseignants-praticiens - à condition de prendre le risque d'un debriefing "à chaud », avec les étudiants. Ce qui est commun aux deux objets, c'est qu'ils restent le point de départ d'un questionnement authentique de la part des enseignants, où ils n'ont pas construit d'avance d'interprétation qui fasse autorité.

Dans tous les cas, aucun des dispositifs proposés ne prend la forme de cours d'histoire ou de sociologie des sciences per se, qui seraient censés toucher par leur vertu propre un public de professionnels : au mieux ils sont complémentaires à ce type de formation. Ainsi, les formations présentées par M. Letté et L. Petitgirard, dans le cadre du CNAM, se composent de plusieurs modules, qui combinent des enseignements pratiques (les Ateliers de médiation numérique discutés dans ce dossier, par exemple) avec des approfondissements plus théoriques (en histoire des sciences, STS, sociologie des sciences). L'expertise universitaire sur les objets historiques et culturels qui entrent en jeu dans la formation, aussi bien que la posture d'enseignants-chercheurs adoptée, sont fondamentalement mises au service d'un dispositif conçu comme centré sur le développement professionnel (cf II. 2). Il faut donc bien concevoir la place de cette expertise comme stratégique dans un dispositif plus global, qui intègre les contraintes du métier. 
27 À l'inverse, les dispositifs pédagogiques peuvent alors être vus comme des espaces d'intéressement (Legros 2008) pour les savoirs EHST ou STS auxquels il s'agit d'initier les étudiants : cet apprentissage d'une démarche de recherche et de questionnement prend en effet sens, dans la mesure où ils deviennent significatifs pour les étudiants parce qu'ils sont reproblématisés sur des objets de leur création ou de leur choix ${ }^{8}$. Cet enjeu est très explicite dans la contribution de M. Letté, car l'objectif d'intéresser les étudiants à la lecture de la littérature STS est un enjeu essentiel.

\section{3. La fonction des espaces dialogiques et de l'écriture collaborative dans l'élaboration d'une créativité}

28 La possibilité offerte aux participants de s'approprier dans un contexte " proximal » des problématiques de recherche est un des éléments clés commun aux dispositifs présentés dans ce volume : à savoir, le fait de prévoir, comme une étape essentielle, des espaces régulés de dialogue qui jouent un rôle essentiel dans l'élaboration des projets ou réflexions de chacun. Ce point est étroitement lié au précédent: si l'expertise des enseignants, et leur posture, ne doivent pas constituer un point de blocage mais bien une ressource pour les participants, c'est parce qu'on prévoit de leur donner la possibilité de penser autrement, d'élaborer pour leur propre compte mais en dialogue avec les autres participants, experts compris.

Que de tels espaces de liberté soient offerts, mais au sein même de la confrontation à la voix d'autrui, est un objet classique de réflexion méthodologique et psychologique pour les recherches qui ont développé différentes méthodes de confrontation à l'activité professionnelle (atelier d'analyse professionnelle, entretien d'explicitation, autoconfrontation, confrontation simple ou croisée). Elles comprennent à titre d'ingrédient ou de complément, des confrontations dialogiques qui autorisent une forme de consensus extérieur (entre pairs) ou intérieur (pour chacun). Chez Clot et ses collaborateurs, qui ont théorisé la confrontation simple et croisée, le fondement théorique de ces recherches repose sur les théories du dialogue de Bakhtine, qui permettent de distinguer différents niveaux de dialogues et de destinataires au sein du même échange (Clot, 2008, p. 203-230). On ne trouvera pas dans ce volume cependant, d'analyse détaillée de tels dialogues; la réflexion des contributeurs porte davantage sur la fonction des espaces dialogiques dans l'analyse comparative et globale de dispositifs de formation'.

Ainsi, et puisque le dialogue est ainsi reconnu comme un espace de confrontation, non seulement aux autres, mais aussi à soi (dissonances intérieures, dilemmes), ou encore à une instance en tiers du dialogue, un "surdestinataire", son déploiement est particulièrement nécessaire aux dispositifs réfléchir-pour-créer. Dans ces derniers en effet, le point d'entrée consiste à installer une possibilité non seulement de divergence de vues ou de lectures, mais encore la possibilité d'un dilemme intérieur entre soi et non soi, comme nous le verrons dans la partie suivante au sujet des espaces transitionnels. En outre, et comme l'indiquent $\mathrm{C}$. Bonah et J. Danet, la référence à un tiers, susceptible d'être analysée en termes de "surdestinataire", est particulièrement importante dans ce processus; de même que la possibilité d'un espace privé de délibération, car ce dernier est la matrice des choix et travaux ultérieurs des étudiants.

31 Dans le cas des dispositifs créer-pour-réfléchir, qui partent d'une démarche de projet centré sur un objectif de création ou de recréation, les espaces réflexifs qui sont ménagés dans ces dispositifs, doivent se penser en lien étroit avec des dispositifs d'échanges et de 
négociation autour des créations concrètes des étudiants. Le dialogisme préconisé est donc, du même coup, difficilement séparable des contraintes propres à l'écriture collaborative, et à la définition d'espaces pour cette dernière - ce qui explique notamment que L. Petitgirard, par exemple, en fasse un concept clé de la démarche de l'Atelier de médiation numérique. Nous reviendrons sur ce point dans la partie suivante, au sujet de la théorie des objets autour desquels se centre alors la réflexion et l'activité collective.

Cette première comparaison critique, portant sur la structure et les éléments clés des dispositifs de formation, nous permet maintenant d'en aborder une seconde, portant sur les grands concepts et cadres théoriques repris et ré-élaborés par les contributeurs-trices, pour penser leurs constructions pédagogiques et leurs enjeux.

\section{Les théorisations possibles de ces dispositifs}

33 Nous résumons et commentons ici les différentes façons dont les enjeux essentiels des dispositifs analysés dans ce volume sont pensés par les contributeurs : en fonction de quels cadres théoriques ou de quelles méthodologies, et plus exactement en fonction de quels détournements de ces derniers. Ces cadres sont principalement empruntés à la psychologie dynamique (objets transitionnels, IV.1), à la gestion de l'innovation (design thinking, IV. 2), enfin à la sociologie de l'innovation (objets frontières et objets intermédiaires, IV. 3). Ce détournement a, dans tous les cas, au moins deux fonctions pour les contributeurs : leur permettre de penser la façon dont on peut ménager un espace de réflexion qui soit ou devienne aussi, pour les participants, un espace de créativité et de formation (cf. III. 3 ci-dessus) ; et de comprendre le rôle que joue ou que peut jouer dans ce travail d'ouverture à la créativité professionnelle, l'expertise en histoire, épistémologie ou sociologie des sciences et techniques (cf. III. 2). Il s'agit donc de reprendre les problématiques introduites précédemment (partie III) mais, pour ainsi dire «vues de l'intérieur", en fonction des principales catégories théoriques mobilisées par les contributeurs-trices, et qui donnent lieu à des échanges explicites entre eux. L'épilogue de L. Chupin offre, en contrepoint de ces théorisations et selon un point de vue issu des sciences de l'information et de la communication, une analyse originale de la fonction des espaces d'écriture et de collaboration numérique, essentiellement dans les dispositifs créer-pour-réfléchir. Cette comparaison critique s'appuie sur son propre travail sur des plateformes collaboratives semblables à celle du Museum d'Histoire Naturelle: Les herbonautes.

\section{1. Espaces transitionnels et objets pré-transitionnels : préparer et accompagner un phénomène transitionnel conduisant à investir de nouveaux objets}

La notion d'objet transitionnel permet d'envisager dans la durée la progression du développement du sujet à la faveur de l'ouverture d'une « aire transitionnelle » où le sujet trouve, et à la fois crée ses objets, dans une sorte de paradoxe fondamental qui conduit Winnicott à parler d'objets trouvés-créés : il les trouve, au sens qu'il y a un effet de réalité, l'objet a été donné au sujet; mais il les crée pourtant, car ce sont ses objets, ceux qu'il investit. Elle a été mobilisée par Bonah et Danet dans leur théorisation du projet de formation conçu autour de la plateforme MEDFILM pour aboutir, par analogie, à ces 
objets transitionnels, à une notion d'outil pédagogique transitionnel. Le film documentaire est pensé comme instance tierce entre étudiants et enseignants, et second point essentiel, il a par lui-même un effet ambigu, renforcé par la nature de l'objet filmique lui-même : il est réel (pour les étudiants, qui perçoivent les situations filmées comme telles) et conditionnel car construit par un cinéaste. Entre étrangeté et identité, l'enseignant vise à induire cette double lecture, à maintenir cette tension et ce paradoxe.

Il reste que le choix des propositions filmiques par l'enseignant est une opération préalable à l'expérience transitionnelle, ce qu'A. Bernard (2016b) propose de définir comme la construction d'objets «pré-transitionnels»: offerts à l'expérience des étudiants, ils donnent potentiellement lieu à des "phénomènes transitionnels ", selon l'expression de Winnicott, qui conduisent les étudiants à investir par eux-mêmes de nouveaux objets. On retrouve le même processus à l'œuvre dans la contribution de M. Letté, mobilisant de manière heuristique des représentations ordinaires des sciences et techniques en société : les premières propositions, en groupes entiers ou par petits groupes, mais toujours sur des objets choisis par les enseignants, conduisent in fine les étudiants à construire et développer un choix d'objets plus personnels, qui donne lieu à une notice sur le carnet. Il en va de même pour les lectures proposées dans le stage de formation continue évoqué par Bernard et Gosztonyi: elles ont une fonction de catalyseur et peuvent déboucher, comme on le voit dans l'exemple proposé, sur un travail autour d'objets directement intéressants pour les collègues, mais qui ne sont pas (ou pas nécessairement) la déclinaison immédiate du matériel étudié. Ce qui compte est plutôt le réinvestissement d'une certaine expérience de lecture.

Cet aspect, même s'il est moins explicite et central que dans les dispositifs réfléchir-pourcréer, reste néanmoins présent dans les aspects préparatoires aux processus de formation créer-pour-réfléchir décrits par M. Guedj et L. Petitgirard : les enseignants sont en effet placés, dans ce cas, en situation d'accompagner une rencontre et une expérience de confrontation à des objets et des dispositifs de médiation préexistants. La constitution d'un choix d'objets patrimoniaux, dans le cadre d'une exposition pensée fondamentalement pour toucher les visiteurs, définit par excellence une aire prétransitionnelle; l'orientation méthodologique, les choix d'espaces à l'intérieur du Musée (investis par les étudiants de l'Atelier de médiation numérique), les sélections d'objets de collections propres au déroulement de l'expérience de l'Atelier de médiation numérique, relèvent d'une problématique similaire et conditionnent la progression des étudiants. Ces problématiques rejoignent les réflexions menées aujourd'hui par les collègues de l'Institut de Recherche et d'Innovation (IRI) du Centre Pompidou sur «l'écosystème muséal » compris comme espaces transitionnels (Drouin Leclerc 2016), au sens d'espaces muséaux dans leur dimension physique, mais qui agrègent une dynamique de participation de la part des visiteurs.

On retrouve dans les réflexions finales de $\mathrm{M}$. Letté, une réflexion proche dans la mesure où, poussé à l'extrême, l'ensemble des objets pré-transitionnels se fond en quelque sorte avec l'ensemble de la culture contemporaine, considérée comme un environnement technoscientifique qui constitue de fait notre culture, comme s'il était tout entier un grand musée. Ce qui compte alors est la prise de conscience, par les étudiants, de ce champ d'investigation possible. Les remarques initiales de C. Bonah et J. Danet sur les fonctions du champ jusqu'ici peu exploré du cinéma utilitaire en médecine et santé, sont très proches: elles ont pour fonction de pousser à reconnaitre l'expertise médicale comme un enjeu partagé dans la société; et par conséquent, pour les étudiants, un 
facteur de réflexion sur les enjeux de leur future profession ainsi que le rôle qu'ils tiendront face aux patients et en société.

\section{2. Détourner le Design thinking vers une écriture collaborative de type réflexif}

Les expériences de type Museomix constituent aujourd'hui des modes d'exploration de pratiques de médiation numérique, s'écartant des propositions descendantes et unilatérales de la vulgarisation: l'Atelier de médiation numérique de L. Petitgirard s'en inspire, tout en détournant ces processus de « remixage » et les méthodologies de design thinking qui les sous-tendent, au profit d'un atelier combinant créativité professionnelle et construction d'une réflexivité en propre. Pour ce faire, le dispositif intègre en son cœur un mode d'écriture collaborative, imposé aux étudiants, sur la forme d'un document numérique partagé. Il a une double fonction : une fonction d'élucidation et de réflexion, en rendant compte par écrit du processus « en train de se faire » (pour chaque groupe en formation), son comment et son pourquoi ; une fonction créative, qui puisse orienter (et surtout faire converger) le groupe en formation vers la production d'un prototype de dispositif de médiation numérique, le plus innovant possible.

Cette double vocation de l'écriture collaborative se trouve également à la marge du processus décrit dans la contribution d'A. Bernard et K. Gosztonyi : écrire pour réfléchir débouche finalement sur l'élaboration des nouvelles ressources pédagogiques et un partage de la réflexion. Ce qui apparaît à la comparaison des deux est une sorte de similitude dans la "récupération » des aspects réflexifs dans la création elle-même : dans un cas, ce sont certains prototypes qui héritent des caractères réflexifs et collaboratifs qui caractérisent le niveau d'écriture collaborative de premier niveau (EC1); dans l'autre, la réflexion donne finalement lieu à une sorte de prototype de ressource pour l'enseignement, elle prend la forme d'une création.

On retrouve probablement là un des enjeux de la «dérive " du modèle du praticien réflexif, issu des réflexions séminales de Schön et devenu depuis hégémonique. Comme le pointent M. Tardif et ses collègues (2012), cette évolution conduit à la limite à décrire ou penser la réflexivité comme une sorte de donnée immanente à l'activité professionnelle elle-même, en dehors de toute friction avec des sphères d'analyse et de savoir extrinsèques à cette activité. Dans le cadre des démarches discutées plus haut, c'est précisément le caractère autarcique et centré sur des procédures d'action (d'innovation en l'occurrence) qui est détourné et enrichi par le contact avec la sphère d'expertise historienne ou STS, pour aboutir à de nouvelles formes qui gardent une trace effective de cet enrichissement. Mais les espaces de réflexion dialogiques qui sont artificiellement ménagés à cet effet, sont en partie des foyers de dissensus épistémologique et restent, à ce titre, fragiles.

41 Il est à cet égard très intéressant de profiter du point de vue " décentré » que propose $\mathrm{L}$. Chupin dans son épilogue, à la lumière d'une analyse de plateformes numériques, dont l'enjeu principal n'est pas la formation d'enseignants ou de médiateurs, mais la valorisation d'un patrimoine scientifique et technique. Or ce que montre son analyse, c'est que les aspects de formation, même s'ils ne sont pas prévus a priori dans ce type de dispositif, font néanmoins naturellement apparition par le jeu des contraintes qui oblige les animateurs des plateformes à organiser le travail avec des novices. On peut alors poser la question, comme le suggère L. Chupin en conclusion, du développement possible d'une 
dimension assumée de formation dans la conception même de ces plateformes. Même si cet aspect est peu développé dans l'étude de M. Letté, on voit ici tout l'intérêt d'étudier la rencontre entre des visées formatrices et pédagogiques, et un jeu de contraintes éditoriales, qui caractérise l'atelier La Lucarne.

\section{3. Objets frontière et intermédiaires : structurer et organiser un dissensus}

La notion d'objet frontière, élaborée au départ dans le champ de la sociologie des sciences, a fait l'objet ensuite de nombreuses réappropriations, dans différents domaines de recherche dépassant la sociologie de l'innovation : elle intéresse notamment le monde de la médiation scientifique et technique ${ }^{10}$ et celui de l'éducation ${ }^{11}$. Dans la critique des usages qui a été faite de ce concept, Vinck et Trompette (2009, p. 13-15) soulignent que l'aspect de "structuration des connaissances" fondamentalement impliquée dans la notion est facilement oublié, et insistent par ailleurs sur l'intérêt d'autres notions voisines et complémentaires, comme celles d'objet intermédiaire qui permet de rendre compte de l'impact organisationnel de certains artefacts dans un travail collectif. Ces deux notions sont ici principalement reprises et détournées par M. Guedj pour servir à une meilleure compréhension des enjeux d'un dispositif de formation d'enseignants à la pédagogie par projet, lui-même inséré dans un ensemble plus vaste visant à valoriser un riche patrimoine universitaire d'instruments et de textes techniques et scientifiques ${ }^{12}$. L'approche vise donc ici, très fondamentalement, à penser le fonctionnement de collectifs imbriqués: à un premier niveau, il s'agit de penser la recherche d'un consensus opératoire dans le collectif des spécialistes réunis pour organiser l'exposition. Cette recherche s'incarne dans l'élaboration d'un document pilote, un objet frontière représentant à la fois un compromis entre les différents objectifs des partenaires, et un élément structurant pour l'organisation effective de l'exposition. À un second niveau, le même document, joint à un cahier des charges, joue le rôle d'objet intermédiaire pour le travail d'un autre ensemble de collectifs: les groupes d'étudiants MEEF qu'on invite à élaborer différents projets de parcours de visite. Les objets en question servent alors à structurer le travail tout en ménageant une «zone de dissensus » indispensable aux objectifs de formation du module.

En fonction de cette analyse de référence, L. Petitgirard propose in fine d'analyser le fonctionnement des espaces collaboratifs intrinsèques à l'Atelier de médiation numérique dans les termes qui s'inspirent directement de cette analyse à deux niveaux : car le point intéressant est ici que les étudiants, tout en devant construire des objets intermédiaires qui organisent leur dissensus, manquent du document pilote que décrit M. Guedj. Comme L. Petitgirard le suggère, on peut alors interpréter l'une des fonctions du dispositif d'écriture collaborative de premier niveau (EC1) comme tenant lieu de cet objet de référence. Cela permet notamment de porter un nouveau regard sur l'enquête ethnographique préliminaire que les étudiants sont invités à construire : le fait qu'elle ne porte pas seulement sur les usages du Musée, mais aussi sur les aléas et contradictions qui ont présidé à la conception muséographique, joue alors manifestement un rôle très important.

D'une façon analogue, l'analyse de M. Guedj pousse A. Bernard et K. Gosztonyi à s'interroger sur la fonction exacte de certains artefacts structurants, comme les schémas permettant d'interpréter des séries de problèmes élaborés par K. Gosztonyi. Autrement 
dit, on voit bien avec cet exemple, dont l'analyse est surtout centrée sur une expérience transitionnelle, comment elle pourrait être relue, non en fonction d'un type générique d'expérience de «rencontres", mais suivant un fonctionnement collectif qui permet à certains des objets structurants pour la recherche sur les corpus de textes historiques, de jouer un rôle dans la réflexion collective d'un groupe d'enseignants. C'est en tout cas un point qui reste à approfondir.

\section{Conclusion}

En outre, plusieurs des dispositifs donnent une place plus ou moins importante à des
outils numériques de valorisation de ces objets et/ou de pratiques collaboratives autour
d'eux. Il est donc tentant, comme le propose L. Chupin dans son article épilogue, de
développer un autre type de comparaison critique qui prend cette fois-ci comme fil rouge,
non plus le potentiel de transformation et de formation des dispositifs sur les
participants, mais le potentiel de transformation ${ }^{14} 14$ des objets à valeur patrimoniale ou
historique concernés directement ou indirectement, dans le cadre de dispositifs faisant
un usage intensif d'outils numériques

En outre, plusieurs des dispositifs donnent une place plus ou moins importante à des
outils numériques de valorisation de ces objets et/ou de pratiques collaboratives autour
d'eux. Il est donc tentant, comme le propose L. Chupin dans son article épilogue, de
développer un autre type de comparaison critique qui prend cette fois-ci comme fil rouge,
non plus le potentiel de transformation et de formation des dispositifs sur les
participants, mais le potentiel de transformation ${ }^{14} 14$ des objets à valeur patrimoniale ou
historique concernés directement ou indirectement, dans le cadre de dispositifs faisant
un usage intensif d'outils numériques

En outre, plusieurs des dispositifs donnent une place plus ou moins importante à des
outils numériques de valorisation de ces objets et/ou de pratiques collaboratives autour
d'eux. Il est donc tentant, comme le propose L. Chupin dans son article épilogue, de
développer un autre type de comparaison critique qui prend cette fois-ci comme fil rouge,
non plus le potentiel de transformation et de formation des dispositifs sur les
participants, mais le potentiel de transformation ${ }^{14} 14$ des objets à valeur patrimoniale ou
historique concernés directement ou indirectement, dans le cadre de dispositifs faisant
un usage intensif d'outils numériques

En outre, plusieurs des dispositifs donnent une place plus ou moins importante à des
outils numériques de valorisation de ces objets et/ou de pratiques collaboratives autour
d'eux. Il est donc tentant, comme le propose L. Chupin dans son article épilogue, de
développer un autre type de comparaison critique qui prend cette fois-ci comme fil rouge,
non plus le potentiel de transformation et de formation des dispositifs sur les
participants, mais le potentiel de transformation ${ }^{14} 14$ des objets à valeur patrimoniale ou
historique concernés directement ou indirectement, dans le cadre de dispositifs faisant
un usage intensif d'outils numériques

En outre, plusieurs des dispositifs donnent une place plus ou moins importante à des
outils numériques de valorisation de ces objets et/ou de pratiques collaboratives autour
d'eux. Il est donc tentant, comme le propose L. Chupin dans son article épilogue, de
développer un autre type de comparaison critique qui prend cette fois-ci comme fil rouge,
non plus le potentiel de transformation et de formation des dispositifs sur les
participants, mais le potentiel de transformation ${ }^{14} 14$ des objets à valeur patrimoniale ou
historique concernés directement ou indirectement, dans le cadre de dispositifs faisant
un usage intensif d'outils numériques

En outre, plusieurs des dispositifs donnent une place plus ou moins importante à des
outils numériques de valorisation de ces objets et/ou de pratiques collaboratives autour
d'eux. Il est donc tentant, comme le propose L. Chupin dans son article épilogue, de
développer un autre type de comparaison critique qui prend cette fois-ci comme fil rouge,
non plus le potentiel de transformation et de formation des dispositifs sur les
participants, mais le potentiel de transformation ${ }^{14} 14$ des objets à valeur patrimoniale ou
historique concernés directement ou indirectement, dans le cadre de dispositifs faisant
un usage intensif d'outils numériques

En outre, plusieurs des dispositifs donnent une place plus ou moins importante à des
outils numériques de valorisation de ces objets et/ou de pratiques collaboratives autour
d'eux. Il est donc tentant, comme le propose L. Chupin dans son article épilogue, de
développer un autre type de comparaison critique qui prend cette fois-ci comme fil rouge,
non plus le potentiel de transformation et de formation des dispositifs sur les
participants, mais le potentiel de transformation ${ }^{14} 14$ des objets à valeur patrimoniale ou
historique concernés directement ou indirectement, dans le cadre de dispositifs faisant
un usage intensif d'outils numériques

Comme nous l'indiquions en introduction, ces derniers exemples indiquent la valeur heuristique qu'on peut prêter à la comparaison critique que nous avons ici résumée. C'est, assez naturellement, en théorisant les enjeux de dispositifs de formation ou de développement professionnels a priori disparates quant à leurs contextes institutionnels, leurs publics et leurs objets, qu'on peut non seulement apercevoir des similitudes possibles, mais aussi interroger les dispositifs à la lumière des concepts qui ont été investis pour en penser d'autres ${ }^{13}$.

On peut par ailleurs remarquer que l'ensemble des dispositifs a un point commun qui n'est discuté en détail que dans la contribution de M. Letté, et dans une certaine mesure dans celle de C. Bonah et J. Danet : c'est qu'ils se fondent sur une condition de possibilité essentielle, qui est l'existence d'un corpus d'objets et de textes suffisamment vaste, soit pour autoriser des procédures de choix (fondamentales pour repérer et étudier des phénomènes transitionnels), soit pour obliger à déterminer des objets structurants au sein d'un collectif qui s'en empare (objets qui peuvent alors être pensés suivant leur fonction pour le collectif).

\section{BIBLIOGRAPHIE}

Aillerie, K. (2015). Le Design Thinking : pour une intégration des TICE dans la scénarisation pédagogique. Site du Ministère de l'Education Nationale (MENSR). https://www.reseaucanope.fr/agence-des-usages/le-design-thinking-pour-une-integration-des-tice-dans-lascenarisation-pedagogique.html (consulté le 28.10.17).

Bensaude-Vincent, B. (2013). L'opinion publique et la science. Paris : La Découverte. 
Bernard, A. (2014). Edition collaborative et médiation des sciences et des techniques : argumentaire détaillé de la table ronde. Carnet de recherche «Sciences et techniques en interférences ", billet 770. http://interferences.hypotheses.org/770 (consulté le 28.10.17).

Bernard, A. (2015). Edition collaborative et médiation des sciences et techniques - ex post. Carnet de recherche « Sciences et techniques en interférences », billet 874. https:// interferences.hypotheses.org/874 (consulté le 28.10.17).

Bernard, A. (2016a). Penser la place des sciences humaines dans la formation des enseignants de sciences et techniques : quels types de recherches possibles? In Recherches en éducation, recherches sur la professionnalisation : consensus et dissensus, éd. Par B. Marin et D. Berger, 217-29. Paris : Réseau nationale des ESPE. http://www.reseau-espe.fr/sites/default/files/ documents/prespe15-bernard.pdf

Bernard, A. (2016b). Note critique sur les « outils pédagogiques transitionnels (Bonah, Danet 2014) ». Carnet de recherche SetT en interférences. https://interferences.hypotheses.org/884

Bernard, A., Dell'Angelo, M., de Montgolfier, S., Godfroy, A.-S., Huchette, M., Mayrargue,

A., et Roux, C. (2014). Les sciences humaines dans les parcours scientifiques et techniques professionnalisants : quelles finalités et quelles modalités pratiques ? Présentation générale. SHS Web of Conferences, 13, 00001. https://doi.org/10.1051/shsconf/20141300001

Bonah, C., et Danet, J. (2014). MedFilm. Enseigner « par et pour la recherche » les SHS en milieu médical à l'aide du film « utilitaire ». In Les sciences humaines dans les parcours scientifiques et techniques professionnalisants : quelles finalités et quelles modalités pratiques ? SHS Web of Conferences, 13, 04001. https://doi.org/10.1051/shsconf/20141304001

Bonneuil, C. et Joly, P.-B. (2013). Sciences, techniques et société. Paris : La Découverte.

Brown, T. (2014). L'Esprit design : Comment le design thinking change l'entreprise et la stratégie. Montreuil (Seine-Saint-Denis) : Pearson Education.

Clot, Y. (2008). Travail et pouvoir d'agir. Paris : PUF.

Drouin-Leclerc, V. (2016). L'écosystème muséal : espaces transitionnels des savoirs. Site de l'Institut de Recherche et d'Innovation du Centre Pompidou.

http://www.iri.centrepompidou.fr/evenement/lecosysteme-museal-espaces-transitionnels-dessavoirs/ (consulté le 28.10.17).

Filâtre, D. (2016). Vers un nouveau modèle de formation tout au long de la vie. Site du Ministère de l'Education Nationale (MENSR).

http://cache.media.education.gouv.fr/file/2016/19/2/

Formation_des_enseignants_Rapport_N6_FTLV_674192.pdf (consulté le 28.10.17)

Las Vergnas, O. (2012). L'institutionnalisation de la « culture scientifique et technique », un fait social français (1970 - 2010). Savoirs, 27, 9-60.

Legros, V. (2008). Pour conjuguer les intérêts professionnels. Recherche et formation 58, 5-10. https://rechercheformation.revues.org/699

Paloque-Berges, C. (2015). Programme et résumés de la journée d'étude « Dispositifs hybrides de négociation du savoir ». Site du Cnam - laboratoire HT2s. http://technique-societe.cnam.fr/3dispositifs-hybrides-de-negociation-du-savoir--708728.kjsp?RH=1393005229497 (consulté le 28.10.17)

Paloque-Berges, C. (2016). Programme et argumentaires de la journée d'étude « Articuler histoire des sciences et des techniques, formation de professionnels et édition en ligne ». Site du Cnam - 
laboratoire HT2s. http://technique-societe.cnam.fr/articuler-histoire-des-sciences-et-destechniques-formation-de-professionnels-et-edition-en-ligne-792173.kjsp?RH=1353591646031 (consulté le 28.10.17).

Rey, B. (2014). La notion de compétence en éducation et formation : enjeux et problèmes. Louvain-la-Neuve, Belgique : De Boeck.

Star, S. L., and Griesemer, J. (1989). Institutional Ecology, « Translations » and Boundary Objects: Amateurs and Professionals in Berkeley's Museum of Vertebrate Zoology, 1907 - 1939. Social Studies of Science, 19, 387-420.

Tardif, M., Borges, C., et Malo, A. (Eds.). (2012). Le virage réflexif en éducation : où en sommesnous 30 ans après Schön? Bruxelles, Belgique : De Boeck.

Trompette, P., et Vinck, D. (2009). Retour sur la notion d'objet-frontière. Revue d'anthropologie des connaissances, 3-1, 5-27.

Vinck, D. (2009). De l'objet intermédiaire à l'objet-frontière : Vers la prise en compte du travail d'équipement. Revue d'anthropologie des connaissances 3, 1-1, 51-72. doi:10.3917/rac.006.0051

\section{NOTES}

1. Société Française d'Histoire des Sciences et des Techniques.

2. Recherche et Formation en Epistémologie et Histoire des Sciences et des Techniques.

3. Voir, à titre d'exemple, le dernier rapport du comité de suivi de la réforme (Filâtre 2016).

4. Voir Bonneuil et Joly, 2013.

5. HASTEC pour Histoire et Anthropologie des Savoirs, des Techniques et des Croyances. Voir https://labexhastec-psl.ephe.fr/

6. Les deux sont du reste centrés sur des objets cibles similaires, à savoir des dispositifs de médiation utilisables dans un contexte muséal, incorporant des objets patrimoniaux.

7. Nous revenons plus loin (IV.3) sur cette notion d'objet frontière et comment elle est convoquée dans les analyses de ce dossier.

8. On le sait, cette notion d'espace d'intéressement a été détourné de la sociologie des sciences vers les questions de formation (Legros 2008, citant Latour et Akrich) : on verra dans la partie suivante comment plusieurs des contributeurs ont repris et détourné des notions de sociologie de l'innovation et/ou des sciences, plus neutres que celle d'espace d'intéressement : celles d'objet frontière et d'objet intermédiaire (Trompette et Vinck, 2009, p.9).

9. Dans une étape ultérieure de l'analyse de ce type de dispositifs, réunir un tel corpus de dialogues serait certainement intéressant et fructueux.

10. Voir les références proposées dans la revue de littérature de Trompette et Vinck (2009,

p. 14) autour notamment des recherches du domaine public understanding of science. Que le monde de la médiation scientifique puisse être lu par ailleurs comme relevant des problématiques de la gestion de l'innovation, est bien expliqué dans la contribution de L. Petitgirard (partie III).

11. Voir les références données dans Trompette et Vinck (2009, p. 15-16).

12. En l'occurrence, la construction collaborative d'une exposition autour du thème de la Lumière.

13. Cette comparaison critique est heuristique au moins à un second titre, également suggéré en introduction : elle nous permet aujourd'hui d'envisager de nouveaux dispositifs de formation et de recherche, sur une base conceptuelle plus sûre. Il est trop tôt ici pour donner de nouveaux exemples. 
14. Transformation ou redocumentarisation, pour reprendre les termes de sciences de l'information introduits par L. Chupin

\section{RÉSUMÉS}

L'histoire des sciences et des techniques dans cinq dispositifs innovants de formation professionnelle : une analyse comparative. Nous proposons ici une comparaison critique de cinq dispositifs de formation professionnelle développés dans des contextes et pour des publics a priori très différents, tous en lien à l'histoire des sciences et des techniques : deux types d'ateliers développés au CNAM en lien avec une formation pour médiateurs scientifiques; le projet MEDFILM développé à l'université de Strasbourg en lien à la formation professionnelle des médecins ; un groupe « histoire et épistémologie » expérimenté à l'IREM de Paris Nord pour des enseignants de mathématiques; enfin un dispositif de formation pour des professeurs des écoles, en lien à l'exposition « Regards croisés sur la lumière » à Montpellier. Au-delà des différences de contexte et de méthodes, nous indiquons leurs points communs et la convergence des outils théoriques mobilisés pour penser ces dispositifs.

We propose a critical comparison of five settings for professional training developed in different contexts and for various audiences, and which are all related to the history of science and technology. These are two workshops developed in CNAM for the training of scientific mediators; the MEDFILM project developed in Strasbourg for the professional training of physicians; a group dealing with the history and epistemology of mathematics, developed in IREM of Paris Nord, for teachers of mathematics; and the training session organized for future teachers at elementary school, organized in Montpellier around the exhibition « Regards croisés sur la lumière ». Beyond the differences in contexts and methods, we point out the common features of these settings and reflect about the theoretical tools used to conceive them.

\section{INDEX}

Mots-clés : histoire des sciences, médiation scientifique, formation professionnelle

Keywords : history of science, professionnal training, science communication

\section{AUTEURS}

\section{ALAIN BERNARD}

Maître de conférences, ESPE de l'académie de Créteil - Centre A. Koyré (UMR 8560) et labex HASTEC (ANR-10-LABX-0085)

\section{LOÏC PETITGIRARD}

Maître de conférences HT2S (CNAM) et labex HASTEC (ANR-10-LABX-0085) 